\title{
Características Morfométricas de las Raíces del Plexo Braquial en el Hombre
}

\author{
Morphometric Characteristics of Brachial Plexus Roots in Man
}

Juan Carlos Torrez Aliendre ${ }^{1,2,3}$ \& Marco Paredes Honorato ${ }^{1,3}$

TORREZ, A. J. C \& PAREDES H. M. Características morfométricas de las raíces del plexo braquial en el Hombre. Int. J. Morphol., 37(3):1187-1191, 2019.

RESUMEN: El plexo braquial se forma a partir de cinco raíces espinales: C5, C6, C7, C8 y T1, proporcionando inervación al miembro superior. Se ha demostrado que el análisis morfométrico de las fibras nerviosas es de gran valor para detectar anormalidades, como el tamaño de los haces nerviosos, el número o tamaño de los axones y el área de mielina. El presente estudio fue realizado en muestras de 10 plexos braquiales ( 5 derechos, 5 izquierdos), obtenidos de 5 cadáveres frescos, de sexo masculino, con un promedio de edad entre $24 \pm 3,14$ (rango, 20-30 años), y $73 \pm$ 5,52 años (rango, 60-90 años), sin antecedentes de neoplasia maligna y patología neuropsiquiátrica. Las muestras fueron fijadas en formalina $10 \%$ y teñidas con hematoxilina-eosina. Los datos obtenidos de los 10 plexos braquiales se agruparon en dos rangos de edades, siendo estos jóvenes y adultos. En el grupo de jóvenes el número de fascículos en $\mathrm{C} 5,4,00 \pm 1,22 ; \mathrm{C} 6,9,25 \pm 3,83 ; \mathrm{C} 7,15,20 \pm 7,57 ; \mathrm{C} 8,8,60 \pm 5,39 ; \mathrm{T} 1,4,80 \pm 3,19$ en adultos el diámetro de la raíz fue C5, 4,77 $\pm 1,31$; C6, 5,39 \pm 0,76; C7, 5,91 \pm 1,63; C8, 5,19 \pm 0,94; T1, 4,86 \pm 1,40. Existen diferencias entre las raíces nerviosas del plexo braquial humano, de individuos jóvenes y adultos chilenos, así el área de la raíz de individuos jóvenes es menor, el número de fascículos decrece en los individuos adultos y sus diámetros siempre son mayores en estos últimos.

PALABRAS CLAVE:Plexo braquial; Raíces; Morfometría.

\section{INTRODUCCIÓN}

El plexo braquial en el Hombre se forma a partir de los ramos anteriores de los nervios espinales C5 a T1, con contribución de $\mathrm{C} 4$ y T2, los cuales proporcionan inervación al miembro superior y por algunos ramos sensitivos y motores a la parte superior de la pared torácica y a estructuras cervicales. Sin embargo, su complejidad es atribuida tanto a su intrincada estructura como a su alta incidencia de variación anatómica (Arad et al., 2013).

El número y la organización de los fascículos dentro de un nervio difieren de uno a otro, entre especies e individuos, y a lo largo de la longitud del nervio. Por lo tanto, no es sorprendente que el debate sobre la organización fascicular continúe en la literatura (Sunderland, 1958; Topp \& Boyd, 2012).

Se ha demostrado que el análisis morfométrico de las fibras nerviosas es de gran valor para detectar anormalidades, como el tamaño de los haces nerviosos, el número o tamaño de los axones y el área de mielina (Li et al., 2013). Los estudios de la estructura de los nervios periféricos en las diferentes edades constituyen una de las direcciones en Neuromorfología (Varsegova, 2013).

Los cambios en el sistema nervioso periférico durante el envejecimiento humano han sido estudiados hace bastante tiempo (Corbin \& Gardner, 1937). Una reducción en la densidad de las fibras mielinizadas con el envejecimiento en los nervios periféricos humanos, ha sido demostrado por diversos investigadores (O'Sullivan \& Swallow, 1968; Cavallotti et al., 2002). Sin embargo, varios estudios no han encontrado ninguna correlación significativa entre la densidad de fibras mielinizadas y la edad (Schellens et al., 1993). Durante el envejecimiento normal, no se produce la pérdida de las células nerviosas, la pérdida neuronal se limita a regiones restringidas del sistema nervioso y es probablemente no más del $10 \%$ (Bonnel, 1984). Sin embargo, se ha reportado deterioro de características morfológicas y

\footnotetext{
${ }^{1}$ Programa de Doctorado en Ciencias Morfológicas, Universidad de La Frontera, Temuco, Chile.

${ }^{2}$ Unidad de Morfología, Departamento de Ciencias Básicas, Universidad de La Frontera, Temuco, Chile.

${ }^{3}$ Laboratorio de Biomoléculas e Inmunidad, Departamento de Ciencias Básicas, Universidad de La Frontera, Temuco, Chile.
} 
funcionales del sistema nervioso periférico asociados al envejecimiento (Kundalic et al., 2014), que se conocen como cambios degenerativos en la estructura del axón y el tejido conectivo que cubre el nervio (Bahcelioglu et al., 2008). Por otra parte, diversos tipos de déficits funcionales pueden ser la consecuencia de una pérdida de fibras nerviosas, anormalidades de la mielina y alteraciones en el tejido conectivo y en la vascularización (Ceballos et al., 1999). De esta manera, relacionado con envejecimiento el área del nervio en sección transversal aumenta con la edad (Cartwright, 2013).

Se ha demostrado que se producen cambios en los nervios periféricos en personas mayores de 60 años. Estos consisten en la reducción de la densidad de la fibra y pérdida de los axones mielinizados y no mielinizados (Jacobs \& Love, 1985). La llamada neuropatía senil se acompaña con frecuencia en la disminución en el número y la densidad de las fibras nerviosas mielinizadas y de numerosas irregularidades en la vaina de mielina (Ugrenovic et al., 2015).

De los cambios estructurales más frecuentes relacionados con la edad está aquel en que los axones disminuyen en número y sus vainas de mielina pueden volverse menos compactas y someterse a una desmielinización segmentaria seguida de remielinización. Algunos investigadores han estudiado el envejecimiento normal del sistema nervioso en otras especies (rata, cobayo, conejo, gato, perro y mono) donde encontraron evidencias de alteraciones morfométricas asociadas con la edad (Vivo, 2007; Pannese, 2011).

De acuerdo a lo señalado previamente son pocos los estudios encontrados en la literatura que hayan realizado un análisis detallado de las características morfológicas y morfométricas de las fibras nerviosas del plexo braquial humano asociado al envejecimiento.

Basado en lo anterior, el objetivo de este trabajo fue analizar algunos parámetros morfométricos en las raíces del plexo braquial humano en individuos jóvenes y adultos, con la finalidad de correlacionar las eventuales diferencias en ambos grupos etarios.

\section{MATERIAL Y MÉTODO}

Material. El estudio fue realizado en muestras obtenidas de los ramos anteriores de las nervios espinales de C5 a T1, provenientes de 10 plexos braquiales ( 5 derechos, 5 izquierdos), obtenidos de 5 cadáveres frescos, de sexo masculino con un promedio de edad entre $24 \pm 3,14$ (rango, 20-30 años), y $73 \pm 5,52$ años (rango, 60-90 años), respectivamente. Las piezas anatómicas fueron procesadas inmediatamente post- mortem. Para ello se utilizó el protocolo de disección descrito por Testut et al. (1966). Al emerger la raíz anterior del plexo por el foramen intervertebral se realizaron dos incisiones, una proximal y otra distal. Las muestras obtenidas $(32 \mathrm{~mm})$, se dividieron en 4 secciones cada una de $8 \mathrm{~mm}$ de longitud.

Procesamiento histológico. Las muestras fueron fijadas en formalina tamponada al $10 \%$ para su análisis histológico e histoquímico. Las muestras fueron disecadas con ayuda de una lupa Ramson (5X). Posteriormente, se incluyeron en parafina, y cortadas en secciones transversales de $7 \mu \mathrm{m}$, con un micrótomo Microm HM 355 E. Finalmente, los cortes se tiñeron con Hematoxilina- Eosina

Medición de diámetro y cálculo de área de las raíces. Se midió el diámetro desde el tejido diseccionado con ayuda de un Digimatic Caliper de precisión 0,05 mm (Mitutoyo). Una vez obtenido este dato se realizó el cálculo del área de la raíz según la fórmula $\operatorname{Pr}^{2}$.

Obtención de imágenes y parámetros morfológicos. Se fotografiaron las placas teñidas con H-E en un microscopio óptico Olympus BX51 (Fig. 1), acoplado a una cámara Leica a distintos aumentos.

Para la medición del área total, diámetro y número de los fascículos se utilizaron fotografías tomadas en microscopio con aumento de 40x y se midieron estos fascículos utilizando el programa Image-Proplus 7 Media Cybernetic. Inc USA., calibrado en milímetros. El conteo del número de fibras totales se realizó en imágenes obtenidas en un área $1 \mathrm{~mm}^{2}$ por fascículo con aumento de $400 \mathrm{X}$.

Análisis estadístico. Se obtuvo el promedio y desviación estándar y se analizaron por medio del software Prisma ${ }^{\circledR} 7.04$ (GraphPad Software, San Diego CA). El análisis de los datos entre los diferentes grupos etarios se realizó usando el test de Mann-Whitney, una prueba estadística no paramétrica. Se compararon las variables para cada una de las raíces.

\section{RESULTADOS}

Análisis de parámetros morfológicos. Los datos se agruparon en la Tabla I. El análisis estadístico para diámetro de raíz, número de fascículos, promedio de área de fascículo, densidad de fibras y número de fibras entre jóvenes y adultos no arrojó diferencias significativas para ninguna de las raíces comparadas. Sin embargo, para el caso del área de la raíz se evidenciaron diferencias significativas en tres de las raíces estudiadas entre ambos grupos etarios, siendo la raíz C5, C7 y C8 las únicas que presentaron diferencias significativas (Fig. 3). 
TORREZ, A. J. C \& PAREDES H. M. Características morfométricas de las raíces del plexo braquial en el Hombre. Int. J. Morphol., 37(3):1187-1191, 2019.

Tabla I. Tabla comparativa del promedio (SD) de parámetros morfométricos estudiados en las raíces del plexo braquial humano de jóvenes (20 a 30 años) y adultos (60 a 90 años). Superíndice representa el grupo etario al cual pertenece. 1 jóvenes; 2 adultos.

\begin{tabular}{lllccccc}
\hline & $\begin{array}{l}\text { Área raíz } \\
\left(\mathrm{mm}^{2}\right)\end{array}$ & $\begin{array}{l}\text { Diámetro } \\
\text { raíz }(\mathrm{mm})\end{array}$ & $\mathrm{N}^{\text {o fascículos }}$ & $\begin{array}{l}\text { Promedio área } \\
\text { fascículos }(\mathrm{mm} 2)\end{array}$ & $\begin{array}{l}\text { Promedio } \\
\text { diámetro de } \\
\text { fascículos }(\mathrm{mm})\end{array}$ & $\begin{array}{l}\text { Densidad de fibras } \\
\text { por mm² }\end{array}$ & $\mathrm{N}^{\text {o de fibra por raíz }}$ \\
\hline $\mathrm{C}^{1}$ & $5,93 \pm 1,48$ & $3,78 \pm 0,98$ & $4,00 \pm 1,22$ & $1,51 \pm 0,73$ & $1,12 \pm 0,28$ & $4918,88 \pm 1858,77$ & $28133,26 \pm 1672,31$ \\
$\mathrm{C} 5^{2}$ & $7,87 \pm 1,78$ & $4,77 \pm 1,31$ & $3,40 \pm 3,83$ & $2,12 \pm 1,17$ & $1,65 \pm 0,74$ & $3388,00 \pm 961,59$ & $11089,10 \pm 2400,69$ \\
$\mathrm{C}^{1}$ & $6,52 \pm 1,57$ & $4,15 \pm 1,05$ & $9,25 \pm 3,83$ & $0,65 \pm 0,41$ & $0,81 \pm 0,31$ & $5587,18 \pm 890,50$ & $27992,53 \pm 13759,22$ \\
$\mathrm{C}^{2}$ & $8,52 \pm 1,13$ & $5,39 \pm 0,76$ & $4,20 \pm 2,32$ & $2,18 \pm 1,15$ & $1,37 \pm 0,57$ & $5059,95 \pm 1341,56$ & $35340,04 \pm 20492,14$ \\
$\mathrm{C}^{1}$ & $8,08 \pm 1,13$ & $5,14 \pm 0,74$ & $15,20 \pm 7,57$ & $0,42 \pm 0,29$ & $0,70 \pm 0,22$ & $5024,17 \pm 879,41$ & $300012,41 \pm 17188,44$ \\
$\mathrm{C} 7^{2}$ & $9,46 \pm 2,73$ & $5,91 \pm 1,63$ & $8,00 \pm 2,76$ & $0,94 \pm 0,40$ & $1,01 \pm 0,25$ & $4414,88 \pm 1722,49$ & $29584,92 \pm 15426,86$ \\
$\mathrm{C} 8^{1}$ & $7,61 \pm 1,55$ & $4,84 \pm 1,03$ & $8,60 \pm 5,39$ & $1,18 \pm 0,66$ & $1,11 \pm 0,43$ & $4692,20 \pm 461,84$ & $28054,69 \pm 4497,48$ \\
$\mathrm{C} 8^{2}$ & $8,91 \pm 2,73$ & $5,19 \pm 0,94$ & $5,80 \pm 3,43$ & $1,93 \pm 1,21$ & $1,22 \pm 0,64$ & $4408,65 \pm 825,84$ & $32676,50 \pm 12200,67$ \\
$\mathrm{~T} 1^{1}$ & $6,76 \pm 2,15$ & $4,31 \pm 1,43$ & $4,80 \pm 3,19$ & $1,62 \pm 1,16$ & $1,38 \pm 0,68$ & $4265,20 \pm 531,03$ & $21184,71 \pm 8281,24$ \\
$\mathrm{~T} 1^{2}$ & $8,26 \pm 2,04$ & $4,86 \pm 1,40$ & $1,60 \pm 0,80$ & $6,25 \pm 5,05$ & $2,69 \pm 1,65$ & $4448,20 \pm 1735,24$ & $34888,60 \pm 22182,04$ \\
\hline
\end{tabular}
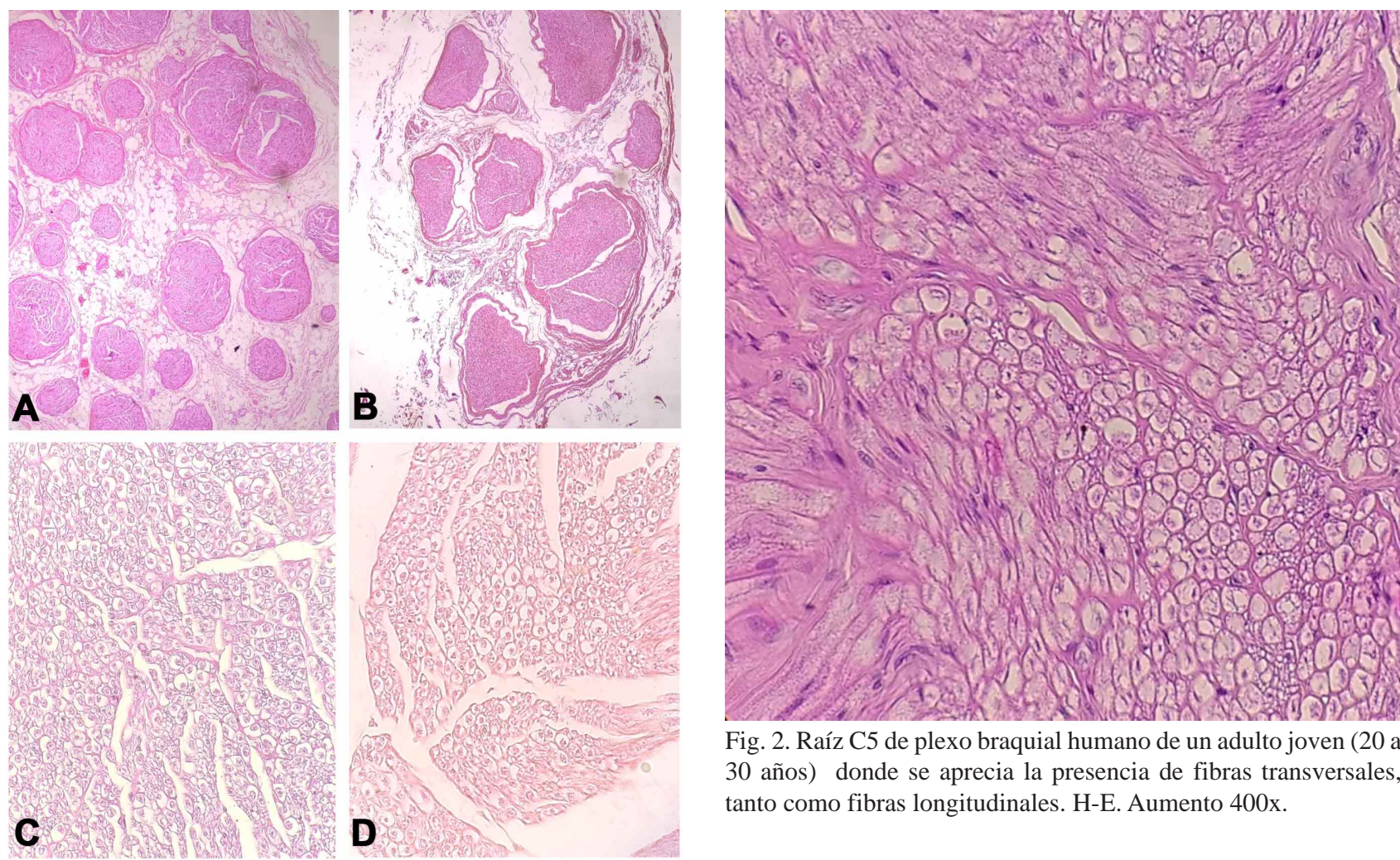

Fig. 2. Raíz C5 de plexo braquial humano de un adulto joven (20 a 30 años) donde se aprecia la presencia de fibras transversales, tanto como fibras longitudinales. H-E. Aumento 400x.

Fig. 1. Raíces de plexo braquial humano de joven (a: 40x, c: 400x) y adulto (b: 40x, d: 400x). H-E
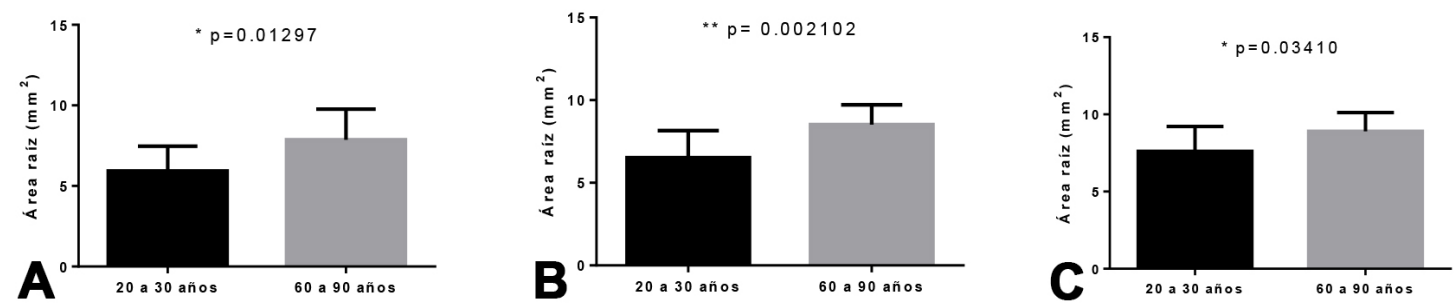

Fig. 3. Representación gráfica del área de las raíces de plexo braquial entre adultos y jóvenes. Raíz C5; p= 0,01297 (a). Raíz C6; p=0,002102 (b). Raíz C5; p=0,03410. 


\section{DISCUSION}

Sobre el plexo braquial existe una abundante literatura en determinadas especies, sin embargo son pocos los estudios que hayan realizado un análisis de las características morfológicas y morfométricas de las fibras nerviosas del plexo braquial humano. Este estudio proporciona datos morfométricos sobre los cambios relacionados con la edad, del plexo braquial, en individuos humanos, jóvenes y adultos.

En nuestro estudio se realizaron mediciones del diámetro del plexo braquial humano en la porción proximal de las raíces, C5, C6, C7, C8 y T1, resultando mayores en los jóvenes que en los adultos (Tabla I), también estos resultados fueron mayores en relación al estudio realizado por Kawai \& Kawabata (1997).

El promedio del diámetro de los fascículos en las cinco raíces del plexo braquial es menor en los individuos jóvenes (Tabla I). Sin embargo, la literatura indica que las raíces del plexo están compuesto por un único fascículo grande (Bonnel). Según Bollini (2004) los fascículos varían de tamaño, de $0.4 \mathrm{~mm}$ a $2 \mathrm{~mm}$, aunque ocasionalmente pueden llegar a medir $4 \mathrm{~mm}$ de diámetro.

Nuestros resultados muestran que las raíces $\mathrm{C5}$, C7, C8, T1, de individuos jóvenes superan en promedio a las raíces de adultos; en la raíz 6 coinciden en el número de fascículos. En C7, el número de fascículos fue consistentemente mayor, lo que probablemente puede significar que desempeña el papel de "Raíz clave" para todo el plexo. Estudios relacionados con el número de fascículos indican dos para $\mathrm{C} 5$, cuatro para $\mathrm{C} 6$, siete para $\mathrm{C} 7$, cuatro para C8, y dos para T1 (Bonnel).

El número de fibras mielínicas halladas en nuestro estudio para el plexo braquial nos indican cifras menores de las raíces C5 y C7, y mayores en C6, C8 y T1 de adultos, en contraposición a los jóvenes.

En esta investigación, el área de la raíz evidenció diferencias significativas en tres de las raíces estudiadas en ambos grupos etarios, siendo las raíces C5, C7 y C8 las que presentaron las diferencias.

En nuestra investigación, el promedio del área de los fascículos fue mayor en las cinco raíces de adultos en relación a los jóvenes. Según Reina et al. (2000), en general, en un determinado nervio el número de fascículos aumenta y el área de cada uno de ellos disminuye en las zonas donde se desprenden las ramas nerviosas y en las zonas próximas a las articulaciones. Por el contrario, el número de los fascículos disminuye y el área de cada uno de ellos aumenta en las zonas ubicadas entre la aparición de las subdivisiones del nervio.

Como se puede apreciar es evidente que existen diferencias entre las raíces nerviosas del plexo braquial humano, de individuos jóvenes y adultos chilenos, donde el área de la raíz de individuos jóvenes es menor, el número de fascículos decrece en los individuos adultos y sus diámetros siempre son mayores en estos últimos.

\section{AGRADECIMIENTOS}

Reconocemos la contribución del personal de Tanatología del Servicio Médico Legal, de Temuco. Unidad de Morfología Departamento de Ciencias Básicas. Laboratorio de Biomoléculas e Inmunidad. Universidad de La Frontera. Temuco, Chile

TORREZ, A. J. C \& PAREDES H. M. Morphometric characteristics of brachial plexus roots in man. Int. J. Morphol., 37(3):1187-1191, 2019.

SUMMARY: The brachial plexus is formed from five spinal roots: $\mathrm{C} 5, \mathrm{C} 6, \mathrm{C} 7, \mathrm{C} 8$ and $\mathrm{T} 1$, providing innervation to the upper limb. It has been shown that the morphometric analysis of nerve fibers is of great value in detecting abnormalities, such as the size of nerve bundles, the number or size of axons and the area of myelin. The present study was performed on samples of 10 brachial plexuses ( 5 right, 5 left), obtained from 5 fresh cadavers, male, with an average age between $24 \pm 3.14$ (range, 20-30 years), and $73 \pm 5.52$ years (range, 60-90 years), with no history of malignancy and neuropsychiatric pathology. The samples were fixed in $10 \%$ formalin and stained with hematoxylineosin. The data obtained from the 10 brachial plexuses were grouped into two age ranges, these being young and adult. In the group of young people the number of fascicles in C5, $4.00 \pm 1.22$; C6, 9.25 \pm 3.83; C7, $15.20 \pm 7.57$; C8, $8.60 \pm 5.39$; T1, $4.80 \pm$ 3.19 in adults the diameter of the root was $\mathrm{C} 5,4.77 \pm 1.31$; 6 , $5.39 \pm 0.76 ; \mathrm{C} 7,5.91 \pm 1.63 ; \mathrm{C} 8,5.19 \pm 0.94 ; \mathrm{T} 1,4.86 \pm 1.40$. There are differences between the nerve roots of the human brachial plexus, young individuals and Chilean adults, so the root area of young individuals is smaller, the number of fascicles decreases in adult individuals and their diameters are always higher in the latter.

KEY WORDS: Brachial plexus; Estate; Morphometry 


\section{REFERENCIAS BIBLIOGRAFICAS}

Arad, E.; Li, Z.; Sitzman, T. J.; Agur, A. M. \& Clarke, H. M. Anatomic sites of origin of the suprascapular and lateral pectoral nerves within the brachial plexus. Plast. Reconstr. Surg., 133(1):20e-7e, 2014.

Bahcelioglu, M.; Elmas, C.; Kurkcuoglu, A.; Calguner, E.; Erdogan, D.; Kadioglu, D. \& Gözil, R. Age-related immunohistochemical and ultrastructural changes in rat oculomotor nerve. Anat. Histol. Embryol., 37(4):279-84, 2008.

Bollini, C. A. Revisión anatómica del plexo braquial. Rev. Argent. Anest., 62(6):386-8, 2004

Bonnel, F. Microscopic anatomy of the adult human brachial plexus: an anatomical and histological basis for microsurgery. Microsurgery, 5(3):107-18, 1984.

Cartwright, M. S.; Mayans, D. R.; Gillson, N. A.; Griffin, L. P. \& Walker, F. O. Nerve cross-sectional area in extremes of age. Muscle Nerve, 47(6):890-3, 2013.

Cavallotti, C.; Pacella, E.; Pescosolido, N.; Tranquilli-Leali, F. M. \& Feher, J. Age-related changes in the human optic nerve. Can. J. Ophthalmol., 37(7):389-94, 2002.

Ceballos, D.; Cuadras, J.; Verdú, E. \& Navarro, X. Morphometric and ultrastructural changes with ageing in mouse peripheral nerve. J. Anat., 195(Pt. 4):563-76, 1999.

Corbin, K. G. \& Gardner, E. D. Decrease in number of myelinated fibers in human spinal roots with age. Anat. Rec., 68(1):63-74, 1937.

Jacobs, J. M. \& Love, V. Qualitative and quantitative morphology of human sural nerve at different ages. Brain, 108(Pt. 4):897-924, 1985.

Kattan, A. E. \& Borschel, G. H. Anatomy of the brachial plexus. J. Pediatr. Rehabil. Med., 4(2):107-11, 2011.

Kawai, H. \& Kawabata, H. Anatomy of the Brachial Plexus. Toh Tuck Lin, World Scientific Publishing, 1997.

Kundalic, B.; Ugrenovic, S.; Jovanovic, I.; Stefanovic, N.; Petrovic, V.; Kundalic, J.; Pavlovic, M. \& Antic, V. Analysis of fascicular structure and connective tissue sheaths in sural nerve during aging. Acta Fac. Med. Naissensis, 31(2):113-20, 2014.

Li, Q.; Xu, D.; He, X.; Wang, Y.; Chen, Z.; Liu, H.; Xu, Q. \& Guo, F. AOTF based molecular hyperspectral imaging system and its applications on nerve morphometry. Appl. Opt., 52(17):3891-901, 2013.

O'Sullivan, D. J. \& Swallow, M. The fibre size and content of the radial and sural nerves. J. Neurol. Neurosurg. Psychiatry, 31(5):464-70, 1968.

Pannese, E. Morphological changes in nerve cells during normal aging. Brain Struct. Funct., 216(2):85-9, 2011.

Reina, M. A.; López Álvarez, A.; de Andrés Ibáñez, J. A. \& Villanueva, M. C. Morfología de los nervios periféricos, de sus cubiertas y de su vascularización. Rev. Esp. Anestesiol. Reanim., 47(10):464-75, 2000.

Schellens, R. L.; van Veen, B. K.; Gabreëls-Festen, A. A.; Notermans, S. L.; van 't Hof, M. A. \& Stegeman, D. F. A statistical approach to fiber diameter distribution in human sural nerve. Muscle Nerve, 16(12):134250, 1993.

Testut, L.; Billet, H.; Dupret, S. \& Jacob, O. Atlas de Disección por Regiones. Barcelona, Salvat, 1966.

Topp, K. S. \& Boyd, B. S. Peripheral nerve: from the microscopic functional unit of the axon to the biomechanically loaded macroscopic structure. J. Hand Ther, 25(2):142-51, 2012.

Ugrenovic', S.; Jovanovic, I.; Vasovic, L.; Kundalic, B.; Cukuranovic, R. \& Stefanovic', V. Morphometric analysis of the diameter and g-ratio of the myelinated nerve fibers of the human sciatic nerve during the aging process. Anat. Sci. Int., 91(3):238-45, 2015.

Varsegova, T. N. Developmental dynamics of morphometric parameters of the tibial nerve in dogs. Neurosci. Behav. Physiol., 43(9):1102-6, 2013.

Vivo, J.; Morales, J. L.; Diz, A.; Galisteo, A. M.; Monterde, J. G.; Agüera, E. \& Miró, F. Estudio morfométrico de las fibras nerviosas del nervio oculomotor del perro. Arch. Med. Vet., 39(1):53-7, 2007

\author{
Dirección para correspondencia: \\ Marco Paredes $\mathrm{H}$. \\ Programa Doctorado en Ciencias Morfológicas \\ Universidad de La Frontera \\ Temuco \\ CHILE
}

Email: marco.paredes@ufrontera.cl

Recibido : 04-03-2019

Aceptado: 06-05-2019 